\title{
Ramsey's coheirs
}

\author{
Eugenio Colla and Domenico Zambella \\ Università di Torino \\ April 2020
}

\begin{abstract}
We use the model theoretic notion of coheir to give short proofs of old and new theorems in Ramsey Theory. As an illustration we start from Ramsey's theorem itself. Then we prove Hindman's theorem and the Hales-Jewett theorem. Finally, we prove two Ramsey theoretic principles that have among their consequences partition theorems due to Carlson and to Gowers.

Msc: primary 05D10, secondary 03C98, 03H99.
\end{abstract}

\section{Introduction}

Ramsey theory has substantial and diverse applications to many parts of mathematics. In particular, Ramsey's theorem has foundational applications to model theory through the Ehrenfeucth-Mostowski construction of indiscernibles and generalizations thereof. In this paper we explore the converse direction, that is, we use model theory to obtain new proofs of classical results in Ramsey Theory.

The Stone-Čech compactification, obtained via ultrafilters, is a widely employed method for proving Ramsey theoretic results. One of its first major applications is the celebrated Galvin-Glazer proof of Hindman's theorem, see e.g. [4]. Our methods are related, but alternative, to the ultrafilter approach. We replace $\beta G$ (the Stone-Čech compactification of a semigroup $G$ ) with a large saturated elementary extension of $G$, i.e. a monster model of $\operatorname{Th}(G / G)$. One immediate advantage is that we work with elements of a natural semigroup with a natural operation. In contrast, elements of $\beta G$ are ultrafilters, that is, sets of sets, and the semigroup operation among ultrafilters is far from straightforward.

This idea is not completely new: in his seminal work on the applications of topological dynamics to model theory [14,15], Newelski replaces the semigroup $\beta G$ with the space of types over $G$ with a suitably defined operation. Our approach is similar, except that, unlike Newelski, we do not pursue connections with topological dynamics, but rather offer an alternative realm of application. The investigation of alternative methods in the study of regularity phenomena has been called for by $\mathrm{Di}$ Nasso [5, Open problem \#1]. This article contains a possible answer.

The model theoretic tools employed in this paper are relatively basic. Section 2 is meant to give an accessible overview of the necessary notions for readers whose expertise is not primarily in model theory. Our results do not require assumptions of model theoretic tameness such as stability, NIP, etc., much like those that use non- 
standard analysis, for example in [6]. Investigating the effect of such assumptions remains as future work.

The second author is grateful to Pierre Simon for suggesting the comparison with nonstandard analysis. Both authors would like to thank Vassilis Kanellopoulos for helpful conversation. When this paper was essentially complete, we became aware of [2], which is worth mentioning since it employs similar methods in a related context.

The paper is divided into two parts. In the first part we prove that the notion of coheir leads to short and elegant proofs of well-known results. Most proofs in this part may be considered folklore, though they have not appeared in the literature so far. They are included here to provide a self-contained, gentle introduction to the techniques that are used in the second part.

As a preliminary illustrative step, we present a proof of Ramsey's theorem (Theorem 3.1). Then we prove a generalization of Hindman's theorem (Theorem 5.1), which is required in the second part of the paper. We also show how to combine Ramsey's and Hindman's theorems in a single proposition - the Milliken-Taylor theorem (Theorem 5.3). Finally, we prove an abstract algebraic version of the HalesJewett theorem (Theorem 6.4) due to Sabine Koppelberg [11].

In the second part of the paper we prove two Ramsey-theoretic properties of semigroups (Lemmas 7.1 and 8.1). As an application, we derive a generalization of Carlson's theorem on colourings of variable words which we present in the style of Koppelberg (Theorem 7.2) and in its classical form (Corollary 7.3). Lemma 8.1 is a partition theorem that generalizes Gowers's FIN $_{k}$ Theorem [8] in a different direction than [12].

The extent of the generalizations mentioned above is limited, and they could be obtained in other ways, but our motivation here is to show the use and relevance of model theoretic methods. Numerous papers in the literature strengthen or generalize the partition theorems considered here. The comparison of the results that appear in these papers is not always straightforward - a few are compared in [1].

The proofs in this paper require a modicum of familiarity with model theory. However, the results can be stated in an elementary language, and in the rest of this introduction we introduce the necessary terminology.

Throughout the paper $G$ is a semigroup and $\Sigma$ a non-empty set of endomorphisms of $G$. For $\bar{a} \in G \leq \omega$ we write

$$
\mathrm{fp}^{\Sigma} \bar{a}=\left\{\sigma_{0} a_{i_{0}} \cdots \cdots \sigma_{k} a_{i_{k}}: i_{0}<\cdots<i_{k}<|\bar{a}|, \bar{\sigma} \in\left(\Sigma \cup\left\{\operatorname{id}_{G}\right\}\right)^{k+1}, k<|\bar{a}|\right\}
$$

Overlined symbols, such as $\bar{a}$ or $\bar{\sigma}$, always denote a tuple, and $a_{i}, \sigma_{i}$ denotes the $i$-th entry of that tuple. 
When $\Sigma$ is empty, we write $\operatorname{fp} \bar{a}$.

1.1 Example For future reference, we instantiate the definition above in the context of free semigroups. Let $G$ be the set of words on a finite alphabet $A \cup\{x\}$, where $x$ is a symbol not in $A$ which we call variable. Let $C$ be the set of words on the alphabet $A$. Words in $C$ are called constant words, while those in $G \backslash C$ are called variable words. When $G$ is endowed with the operation of concatenation of words, $C$ and $G \backslash C$ are subsemigroups of $G$. For $t \in G$ and $a \in A$, let $t(a)$ be the word obtained by replacing all the occurrences of $x$ in $t$ by $a$. Note that the map $\sigma_{a}$ : $t \mapsto t(a)$ is an endomorphism of $G$. In the literature, when $G$ is as above and $\Sigma=\left\{\sigma_{a}: a \in A\right\}$, the elements of $\operatorname{fp}^{\Sigma} \bar{s}$ are called extracted words. We say that a tuple $\bar{a} \in\left(\mathrm{fp}^{\Sigma} \bar{s}\right)^{\omega}$ is an extracted sequence if $a_{i} \in \mathrm{fp}^{\Sigma \mathcal{S}_{\Gamma\left[n_{i}, n_{i+1}\right)}}$ for some increasing sequence of positive integers $\left\langle n_{i}: i<\omega\right\rangle$. If, moreover, $a_{i} \notin C$ for all $i$, we say that $\bar{a}$ is an extracted variable sequence of $\bar{s}$.

The following definition will be used to express our results in the general context of semigroups.

1.2 Definition Let $<$ be a binary relation on $G$. We say that $G$ is <-covered if for every finite $A \subseteq G$ there is a $c$ such that $A<c$. If $c$ can be found in some fixed $B \subseteq G$, we say $<$-covered by $B$. We say that $G$ is $<-$ closed if $a<b<c$ implies $a<b \cdot c$ for all $a, b, c \in G$. $A<$-chain in $G$ is a tuple $\bar{a} \in G^{\leq \omega}$ such that $a_{i}<a_{i+1}$.

The preorder relation given by the length of the words on a free semigroup $G$ is a natural example that is both $<$-closed and $<$-covered. A less straightforward relation is used in the proof of Theorem 7.2.

Finally, we recall two standard notions. Let $C \subseteq G$ be a subsemigroup. We say that $C$ is nice if $a \cdot b \in C$ implies $a, b \in C$. A homomorphism $\sigma: G \rightarrow C$ such that $\sigma_{\lceil C}=\mathrm{id}_{C}$ is called retraction of $G$ onto $C$. Note that the set of constant words in Example 1.1 is a nice subsemigroup and that the maps $\sigma_{a}$ are retractions.

We are now ready to state Lemma 7.1.

Lemma Let $\Sigma$ be a finite set of retractions of $G$ onto a nice subsemigroup $C$. Let $<$ be a relation on $G$ that makes it $<$-closed and $<$-covered by $G \backslash C$. Then, for every finite coloring of $G$, there is a $<$-chain $\bar{a} \in(G \backslash C)^{\omega}$ such that $\operatorname{fp}^{\Sigma} \bar{a} \backslash C$ is monochromatic.

When $C$ and $\Sigma$ are empty and $<$ holds for all pairs, the lemma reduces to Hindman's theorem (Theorem 5.1).

The appropriate choice of $G, C, \Sigma$ and $<$ yields Carlson's partition theorem (in particular no model theoretic argument is necessary, see Theorem 7.2 and its Corollary 7.3).

In the last section we prove Lemma 8.1 which is similar to the lemma above but 
deals with composition of homomorphisms. This is also stated in an elementary language and a general version of a partition theorem by Gowers is derived from it.

\section{Coheirs, and coheir sequences}

We assume that the reader is familiar with undergraduate model theory and in this section we only review the few prerequisites that go beyond that. Proofs are omitted. The reader may consult any standard model theory textbook e.g. [18] (the intrepid reader may consult [19], some lecture notes which use the same notation and quirks as this paper). The notation and terminology are standard with the possible exception of Definitions 2.3 and 2.5.

A sequence is a function whose domain is a linear order. A tuple is a sequence whose domain is an ordinal. The domain of the tuple $c$ is denoted by $|c|$ and is called the length of $c$.

2.1 Notation Sometimes (i.e. not always) we may overline tuples as mnemonic. When a tuple $\bar{c}$ is introduced, $c_{i}$ denotes the $i$-th element of $\bar{c}$. We write $c_{\mid I}$, where $I \subseteq|\bar{c}|$, for the tuple which is naturally associated to the restriction of $\bar{c}$ to $I$. The bar is dropped for ease of notation.

We denote the monster model by $\mathcal{U}$ or, when dealing with semigroups, by $\mathcal{G}$. We always work over a fixed set of parameters $A \subseteq \mathcal{U}$. When this set is a model, as it will often be, we denote it by $M$, or $G$ in the case of semigroups.

We say that a type $p(x)$ is finitely satisfied in $A$ if every conjunction of formulas in $p(x)$ has a solution in $A^{|x|}$. A global type that is finitely satisfiable in $A$ is invariant over $A$.

If $M$ is a model every consistent type $p(x) \subseteq L(M)$ is finitely satisfied in $M$. For this reason in a few points in this paper it is necessary to work over a model. For simplicity, we always assume this.

The following is an easy, well-known fact.

2.2 Proposition Every type $q(x) \subseteq L(\mathcal{U})$ that is finitely satisfiable in $M$ has an extension to a global type finitely satisfiable in $M$.

If $p(x)$ is finitely satisfied in $M$, the extensions of $p(x)$ that are also finitely satisfied in $M$ are called coheirs of $p(x)$.

In many cases it is useful to focus on elements instead of their types. We introduce the following notation to express that $\operatorname{tp}(a / M, b)$ is finitely satisfied in $M$. (The notion is standard in model-theory, it has no standard notation though.)

2.3 Definition For every $a \in \mathcal{U}^{|x|}$ and $b \in \mathcal{U}^{|z|}$ we define

$$
a I_{M} b \Leftrightarrow \varphi(a ; b) \text { for all } \varphi(x ; z) \in L(M) \text { such that } M^{|x|} \subseteq \varphi\left(U^{|x|} ; b\right)
$$


We call this the coheir-heir relation. We define the type

$$
x \mathcal{I}_{M} b=\left\{\varphi(x ; b): \varphi(x ; b) \in L(M) \text { and } M^{|x|} \subseteq \varphi\left(U^{|x|} ; b\right)\right\} .
$$

The tuples a realizing this type are those such that $a I_{M} b$. We will use the symbol $a \equiv_{A} x I_{M} b$ for the union of the types $x I_{M} b$ and $\operatorname{tp}(a / M)$.

We imagine $a I_{M} b$ as saying that $a$ is independent from $b$ over $M$. This is a very strong form of independence. In general it is not symmetric, that is, $a I_{M} b$ is not the same as $b I_{M} a$ (symmetry is equivalent to stability).

We shall use, sometimes without reference, the following easy lemma.

2.4 Lemma The following properties hold for all small $M, a, b$, and $c$

1. $a I_{M} b \Rightarrow f a I_{M} f b$ for every $f \in \operatorname{Aut}(U / M)$

invariance

2. $a I_{M} b \Leftrightarrow a_{0} I_{M} b_{0}$ for all finite $a_{0} \subseteq a$ and $b_{0} \subseteq b$

finite character

3. $a I_{M} b, c$ and $b I_{M c} \Rightarrow a, b I_{M} c$

transitivity

4. $a I_{M} b \Rightarrow$ there exists $a^{\prime} \equiv_{M, b}$ a such that $a^{\prime} I_{M} b, c$

coheir extension

Note that $a \equiv_{M} x I_{M} b$ is the intersection of all types in $S(M, b)$ that are coheirs of $\operatorname{tp}(a / M)$. As there may be more than one of such coheirs, $a \equiv_{M} x \beth_{M} b$ need not be a complete over $M, b$. In fact, completeness is a rather strong property.

2.5 Definition If $a \equiv_{M} x I_{M} b$ is a complete type (over $M, b$ ) for every a $\in \mathcal{U}^{<\omega}$, every $b \in U|x|$, and every tuple of variables $x$, then we say that $\mathcal{I}_{M}$ is stationary. We say $n$-stationary if the requirement above is restricted to $|x|=n$.

Stationarity is often ensured by the following property.

2.6 Proposition Fix a tuple of variable $x$ of length $n$. If for every $\varphi(x) \in L(\mathcal{U})$ there is a formula $\psi(x) \in L(M)$ such that $\varphi\left(M^{|x|}\right)=\psi\left(M^{|x|}\right)$ then $I_{M}$ is n-stationary.

2.7 Remark Stationarity of $\mathcal{I}_{M}$ over every model $M$ is equivalent to the stability of $T$. However, in unstable theories the assumption may hold for some particular model. For example, if every subset of $M^{n}$ is the trace of a definable set, then $\nearrow_{M}$ is $n$ stationary by the proposition above. This simple observation will be of help in the proof of Theorem 5.1. For natural example let $T=T_{\mathrm{dlo}}$ and let $M \subseteq \mathcal{U}$ have the order-type of $\mathbb{R}$. By quantifier elimination every definable of $U$ is union of finitely many intervals. By Dedekind completeness, the trace on $A$ of any interval of $U$ coincides with that of an $M$-definable interval.

Let $p(x) \in S(\mathcal{U})$ be a global type that is finitely satisfiable in $M$. We say that the tuple $\bar{c}$ is a coheir sequence of $p(x)$ over $M$ if for every $i<|\bar{c}|$

$$
c_{i} \vDash p_{\left\lceil M, c_{\mid i}\right.}(x) .
$$


The following is a convenient characterization of coheir sequences.

2.8 Lemma For $\bar{c}$ a tuple of length $\omega$, the following are equivalent

1. $\quad \bar{c}$ is a coheir sequence over $M$;

2. $c_{n} \mathfrak{I}_{M} c_{\mid n}$ and $c_{n+1} \equiv_{M, c_{\mid n}} c_{n}$ for every $n<\omega$.

Let $I,<_{I}$ be a linear order. We call a function $\bar{a}: I \rightarrow U|x|$ an $I$-sequence, or simply a sequence when $I$ is clear.

If $I_{0} \subseteq I$ we call $a_{\Gamma I_{0}}$, the restriction of $\bar{a}$ to $I_{0}$, a subsequence of $\bar{a}$. When $I_{0}$ is finite we identify $a_{\uparrow I_{0}}$ with a tuple of length $\left|I_{0}\right|$.

2.9 Definition Let $I,<_{I}$ be an infinite linear order and let $\bar{a}$ be an I-sequence. We say that a is a sequence of indiscernibles over $A$ or, a sequence of $A$-indiscernibles, if $a_{\uparrow I_{0}} \equiv_{A} a_{\Gamma_{1}}$ for every $I_{0}, I_{1} \subseteq$ I of equal finite cardinality.

The following can be easily derived from the lemma above by induction.

2.10 Proposition Every sequence of coheirs over $M$ is M-indiscernible.

\section{Ramsey's theorem from coheir sequences}

We illustrate the relation between coheirs and Ramsey phenomena in the simplest possible case: Ramsey's theorem. The subsequent sections build on this proof for more sophisticated results.

In this chapter we deal with finite partitions. The partition of a set $X$ into $k$ subsets is often represented by a map $f: X \rightarrow[k]$. The elements of $[k]=\{1, \ldots, k\}$ are also called colors, and the partition a coloring, or $k$-coloring, of $X$. We say that $Y \subseteq X$ is monochromatic if $f$ is constant on $Y$.

Let $M$ be an arbitrary infinite set. Fix $n, k<\omega$ and fix a coloring $f$ of the set of all $n$-subsets of $M$, aleas the complete $n$-uniform hypergraph with vertex set $M$,

$$
f:\left(\begin{array}{c}
M \\
n
\end{array}\right) \rightarrow[k] .
$$

We say that $H \subseteq M$ is a monochromatic subgraph if the subgraph induced by $H$ is monochromatic. In the literature monochromatic subgraphs are also called homogeneous sets.

The following is a very famous theorem which we prove here in an unusual way. The proof will serve as a blueprint for other constructions in this paper.

3.1 Ramsey's Theorem Let $M$ be an infinite set. Then for every positive integer $n$ and every finite coloring of the complete n-uniform hypergraph with vertex set $M$ there is an infinite monochromatic subgraph. 
Proof Let $L$ be a language that contains $k$ relation symbols $r_{1}, \ldots, r_{k}$ of arity $n$. Given a $k$-coloring $f$ we define a structure with domain $M$. The interpretation of the relation symbols is

$$
r_{i}^{M}=\left\{a_{1}, \ldots, a_{n} \in M: f\left(\left\{a_{1}, \ldots, a_{n}\right\}\right)=i\right\} .
$$

We may assume that $M$ is an elementary substructure of some large saturated model $\mathcal{U}$. Pick any type $p(x) \in S(\mathcal{U})$ finitely satisfied in $M$ but not realized in $M$ and let $\bar{c}=\left\langle c_{i}: i\langle\omega\rangle\right.$ be a coheir sequence of $p(x)$.

There is a first-order sentence saying that the formulas $r_{i}\left(x_{1}, \ldots, x_{n}\right)$ are a coloring of $\left(\begin{array}{c}M \\ n\end{array}\right)$. Then by elementarity the same holds in U. By indiscernibility, all tuples of $n$ distinct elements of $\bar{c}$ have the same color, say 1 . We now prove that there is a sequence $\bar{a}=\left\langle a_{i}: i<\omega\right\rangle$ in $M$ with the same property.

We construct $a_{\lceil i}$ by induction on $i$ as follows.

Assume as induction hypothesis that the subsequences of length $n$ of $a_{\uparrow i}, c_{\lceil n}$ all have color 1. Our goal is to find $a_{i} \in M$ such that the same property holds for $a_{\uparrow i}, a_{i}, c_{\uparrow n}$. By the indiscernibility of $\bar{c}$, the property holds for $a_{\mid i}, c_{\mid n}, c_{n}$. And this can be written by a formula $\varphi\left(a_{\uparrow i}, c_{\lceil n}, c_{n}\right)$. As $\bar{c}$ is a coheir sequence, by Lemma 2.8 we can find $a_{i} \in M$ such that $\varphi\left(a_{\lceil i}, c_{\lceil n}, a_{i}\right)$. So, as the order is irrelevant, $a_{\lceil i}, a_{i}, c_{\lceil n}$ satisfies the induction hypothesis.

\section{Idempotent orbits in semigroups}

In this and the following sections we fix a semigroup $G$ which we identify with a first-order structure. The language contains, among others, the symbol · which is interpreted as a binary associative operation on $G$. We write $\mathcal{G}$ for a large saturated elementary extension of $G$.

For any two sets $\mathcal{A}, \mathcal{B} \subseteq \mathcal{G}$ we define

$$
\mathcal{A} \cdot G \mathcal{B}=\left\{a \cdot b: a \in \mathcal{A}, b \in \mathcal{B} \text { and } a I_{G} b\right\}
$$

In this and the next section we abbreviate $\mathcal{O}(a / G)$, the orbit of $a$ under $\operatorname{Aut}(\mathcal{G} / G)$, with $a_{G}$. We write $a \cdot{ }_{G} \mathcal{B}$ for $\mathcal{O}(a / G) \cdot{ }_{G} \mathcal{B}$. Similarly for $\mathcal{A} \cdot{ }_{G} b$ and $a \cdot{ }_{G} b$.

4.1 Lemma If $\mathcal{A}$ is type definable over $G$ then so is $\mathcal{A} \cdot{ }_{G} b$ for any $b$.

Proof The set $\mathcal{A} \cdot{ }_{G} b$ is the union of $\mathcal{A} \cdot{ }_{G}\{c\}$ as $c$ ranges in $b_{G}$. The set $\mathcal{A}{ }_{G}\{c\}$ is type definable, say by the the type $\exists y p(x, y, c)$ where

$$
p(x, y, c)=y I_{G} c \wedge y \cdot c=x \wedge y \in \mathcal{A}
$$

Note that, by the invariance of $\beth_{G}$, if $f \in \operatorname{Aut}(\mathcal{G} / G)$, then $\exists y p\left(x, y, f_{c}\right)$ defines $\mathcal{A} \cdot G\left\{f_{c}\right\}$. Therefore if $q(z)=\operatorname{tp}(b / G)$ then $\exists y, z[q(z) \cup p(x, y, z)]$ defines $\mathcal{A} \cdot{ }_{G} b$.

By the invariance of $\mathcal{I}_{G}$, for every $f \in \operatorname{Aut}(\mathcal{G} / G)$ we have $f\left[\mathcal{A} \cdot{ }_{G} \mathcal{B}\right]=f[\mathcal{A}] \cdot{ }_{G} f[\mathcal{B}]$. Therefore when $\mathcal{A}$ and $\mathcal{B}$ are invariant over $G$, also $\mathcal{A} \cdot{ }_{G} \mathcal{B}$ is invariant over $G$. Below 
we mainly deal with invariant sets.

4.2 Proposition For all G-invariant sets $\mathcal{A}, \mathcal{B}$, and $\mathcal{C}$

$$
\mathcal{A} \cdot{ }_{G}\left(\mathcal{B} \cdot{ }_{G} \mathrm{C}\right) \subseteq(\mathcal{A} \cdot G \mathcal{B}) \cdot G \mathrm{C} .
$$

Proof Let $a \cdot b \cdot c$ be an arbitrary element of the 1.h.s. where $a I_{G} b \cdot c$ and $b I_{G} c$. By extension (Lemma 2.4), there exists $a^{\prime}$ such that $a \equiv_{G, b \cdot c} a^{\prime} I_{G} b \cdot c, b, c$. By transitivity (again Lemma 2.4), $a^{\prime} \cdot b I_{G} c$. Therefore $a^{\prime} \cdot b \cdot c$ belongs to the r.h.s. Finally, as $a^{\prime} \equiv_{G, b \cdot c} a$, also $a \cdot b \cdot c$ belongs to the r.h.s. by invariance.

Let $\mathcal{A}$ be a non-empty set. When $\mathcal{A} \cdot{ }_{G} \mathcal{A} \subseteq \mathcal{A}$, we say that it is idempotent (over $G$ ).

4.3 Corollary Assume $\mathcal{B} \subseteq \mathcal{A}$ are both $G$-invariant. Then if $\mathcal{A}$ is idempotent, also $\mathcal{A} \cdot{ }_{G} \mathcal{B}$ is idempotent.

Proof We check that if $\mathcal{A}$ is idempotent so is $\mathcal{A} \cdot{ }_{G} \mathcal{B}$

$$
\begin{array}{rlrl}
\left(\mathcal{A} \cdot{ }_{G} \mathcal{B}\right) \cdot{ }_{G}\left(\mathcal{A} \cdot{ }_{G} \mathcal{B}\right) & \subseteq \mathcal{A} \cdot{ }_{G}\left(\mathcal{A} \cdot{ }_{G} \mathcal{B}\right) & & \text { because } \mathcal{A} \cdot{ }_{G} \mathcal{B} \subseteq \mathcal{A} \\
& \subseteq(\mathcal{A} \cdot G \mathcal{A}) \cdot{ }_{G} \mathcal{B} & & \text { by the lemma above } \\
& \subseteq \mathcal{A} \cdot G \mathcal{B} &
\end{array}
$$

We show that, under the assumption of stationarity, the operation ${ }_{G} G$ is associative. The quotient map $\mathcal{G} \rightarrow \mathcal{G} / \equiv_{G}$ is almost a homomorphism.

4.4 Proposition Assume $I_{G}$ is 1-stationary, see Definition 2.5. Fix a $I_{G} b$ arbitrarily. Then $a^{\prime} \cdot b^{\prime} \equiv_{G} a \cdot b$ for every $a^{\prime} \equiv_{G} a$ and $b^{\prime} \equiv_{G} b$ such that $a^{\prime} I_{G} b^{\prime}$. Or, in other words,

$$
(a \cdot b)_{G}=a \cdot G b .
$$

Proof We prove two inclusions, only the second one requires stationarity.

$\subseteq$ As $a I_{G} b$ holds by hypothesis, $a \cdot b \in a \cdot G b$. The inclusion follows by invariance.

$\supseteq$ By invariance it suffices to show that the 1.h.s. contains $a \cdot{ }_{G}\{b\}$. Let $a^{\prime} \in a_{G}$ such that $a^{\prime} I_{G} b$. We claim that $a^{\prime} \cdot b \in(a \cdot b)_{G}$. Both $a$ and $a^{\prime}$ satisfy $a \equiv_{G} x \beth_{G} b$. By 1-stationarity, $a \equiv_{G, b} a^{\prime}$. Hence $a \cdot b \equiv_{G} a^{\prime} \cdot b$.

4.5 Corollary (associativity) Assume $I_{G}$ is 1-stationary. Then for all G-invariant sets $\mathcal{A}$, $B$ and $\mathcal{C}$

$$
\mathcal{A} \cdot{ }_{G}\left(\mathcal{B} \cdot{ }_{G} \mathrm{C}\right)=\left(\mathcal{A} \cdot{ }_{G} \mathcal{B}\right) \cdot{ }_{G} \mathrm{C} \text {. }
$$

Proof We can assume that $\mathcal{A}, \mathcal{B}$ and $C$ are $G$-orbits. Say of $a, b$, and $c$ respectively. We can assume that $a I_{G} b \cdot c$ and $b I_{G} c$. By Proposition 4.4 the set on the 1.h.s. equals $(a \cdot b \cdot c)_{G}$. By a similar argument the set on the r.h.s. equals $\left(a^{\prime} \cdot b^{\prime} \cdot c^{\prime}\right)_{G}$ for some elements $a^{\prime}, b^{\prime}$, and $c^{\prime}$. Proposition 4.2 proves that inclusion $\subseteq$ holds in general. But inclusion between orbits amounts to equality.

The following lemma proves the existence of idempotent orbits. The proof is selfcontained, i.e. it does not use Ellis's theorem on the existence of idempotents in 
compact left topological semigroups (however, the argument is very similar). As a comparison, finding a proof in the setting of nonstandard analysis is listed as an open problem in [5].

4.6 Lemma Assume $\mathcal{I}_{G}$ is 1-stationary. If $\mathcal{A}$ is minimal among the idempotent sets that are type-definable over $G$, then $\mathcal{A}=b_{G}$ for some (any) $b \in \mathcal{A}$.

Proof Fix arbitrarily some $b \in \mathcal{A}$. By Corollary 4.3 , the set $\mathcal{A} \cdot{ }_{G} b$ is contained in $\mathcal{A}$, idempotent and type-definable over $G$ by Lemma 4.1 . Therefore by minimality $\mathcal{A} \cdot{ }_{G} b=\mathcal{A}$. Let $\mathcal{A}^{\prime} \subseteq \mathcal{A}$ be the set of those $a$ such that $a \cdot{ }_{G} b=b_{G}$. This set is non-empty because $b \in \mathcal{A} \cdot{ }_{G} b$. It is easy to verify that $\mathcal{A}^{\prime}$ is type-definable over $G, b$. As it is clearly invariant over $G$, it is type-definable over $G$. By associativity it is idempotent. Hence, by minimality, $\mathcal{A}^{\prime}=\mathcal{A}$. Then $b \in \mathcal{A}^{\prime}$, which implies $b \cdot{ }_{G} b=b_{G}$. That is, $b$ has idempotent orbit. Finally, by minimality, $\mathcal{A}=b_{G}$.

4.7 Corollary Under the same assumptions of the lemma above, every idempotent set that is type-definable over $G$ contains an element with an idempotent orbit.

\section{Hindman's theorem}

In this section we merge the theory of idempotents presented in Section 4 with the proof of Ramsey's theorem to obtain Hindman's theorem.

Let $\bar{a}$ be a tuple of elements of $G$ of length $\leq \omega$. In Section 1 we defined fp $\bar{a}$ and the notions of $<$-closed and $<$-covered. The relation $<$ is introduced mainly for future reference. The classical Hindman's theorem is obtained with the positive integers (as an additive semigroup) for $G$ and $<$ for $<$.

5.1 Hindman Theorem Let $<$ be a relation on $G$ that makes it $<-$-closed and $<-$ covered. Then for every finite coloring of $G$ there is $a<-$-chain $\bar{a}$ such that $\mathrm{fp} \bar{a}$ is monochromatic. If there is no $g \in G$ such that $G<g$, we may further assume that the elements of the $<$-chain are all distinct.

Proof We interpret $G$ as a structure in a language that extends the language of semigroups with a symbol for $<$ and one for each subset of $G$. Let $\mathcal{G}$ be a saturated elementary superstucture of $G$. As observed in Remark 2.7, the language makes $\beth_{G}$ trivially 1-stationary.

We write $\mathcal{G}^{\prime}$ for the type-definable set $\{g: G<g\}$, which is non-empty because $G$ is $<$-covered. We claim that $\mathcal{G}^{\prime}$ is idempotent. In fact, if $a, b \in \mathcal{G}^{\prime}$ then, as $G<a, b$ and $a I_{G} b$, we must have that $a<b$. Therefore, from the $<$-closedness of $G$ we infer $a \cdot b \in \mathcal{G}^{\prime}$.

Let $g_{0}$ be an element of $\mathcal{G}^{\prime}$ with idempotent orbit as given by Corollary 4.7. We can assume that $g_{0} \notin G$ otherwise the sequence that is identically $g_{0}$ trivially proves the theorem. If we want the elements of the chain $\bar{a}$ to be distinct it suffices require that 
$g_{0} \notin G$. By definition of $g_{0}$, this can be directly assumed when there is no $g \in G$ such that $G<g$. Let $p(x) \in S(\mathcal{G})$ be a global coheir of $\operatorname{tp}\left(g_{0} / G\right)$. Let $\bar{g}$ be a coheir sequence of $p(x)$, that is

$$
g_{i} \vDash p_{\left\lceil G, g_{\mid i}\right.}(x) .
$$

We write $\overleftarrow{g}_{\mid i}$ for the tuple $g_{i-1}, \ldots, g_{0}$. By the idempotency of $\left(g_{0}\right)_{G}$ and Proposition $4.4, h \equiv_{G} g_{0}$ for all $h \in \mathrm{fp} \overleftarrow{g}_{\mid i}$ and all $i$. It follows in particular that fp $\overleftarrow{g}_{\uparrow i}$ is monochromatic, say all its elements have color 1 . Now, we use the sequence $\bar{g}$ to define $\bar{a} \in G^{\omega}$ such that all elements of fp $\bar{a}$ have color 1 .

Assume as induction hypothesis that $\operatorname{fp}\left(a_{\uparrow i}, g_{0}\right)$ is monochromatic of color 1 . Our goal is to find $a_{i}$ such that the same property holds for $\mathrm{fp}\left(a_{\mid i+1}, g_{0}\right)$.

First we claim that from the induction hypothesis it follows that, for all $j$, all elements of $\operatorname{fp}\left(a_{\mid i}, \overleftarrow{g}_{\mid j}\right)$ have color 1 . In fact, the elements of $\operatorname{fp}\left(a_{\uparrow i}, \overleftarrow{g}_{\mid j}\right)$ have the form $b \cdot h$ for some $b \in \operatorname{fp}\left(a_{\lceil i}\right)$ and $h \in \mathrm{fp}\left(\overleftarrow{g}_{\lceil j}\right)$. As $h \equiv_{G} g_{0}$, we conclude that $b \cdot h \equiv_{G} b \cdot g_{0}$, which proves the claim.

Let $\varphi\left(a_{\mid i}, g_{i+1}, g_{\mid i+1}\right)$ say that all elements of $\operatorname{fp}\left(a_{\mid i}, \overleftarrow{g}_{\mid i+2}\right)$ have color 1 . As $\bar{g}$ is a coheir sequence we can find $a_{i}$ such that $\varphi\left(a_{\uparrow i}, a_{i}, g_{\lceil i+1}\right)$. Hence all elements of $\mathrm{fp}\left(a_{\mid i+1}, \overleftarrow{g}_{\mid i+1}\right)$ have color 1 . Therefore $a_{i}$ is as required.

Hindman's theorem generalizes to a proposition that subsumes Ramsey's theorem. It is usually referred to as the Milliken-Taylor theorem [13] and [17]. By the following observation, we may use virtually the same proof.

5.2 Proposition Assume $\mathfrak{I}_{G}$ is 1-stationary. Let $\bar{g} \in \mathcal{G}^{\omega}$ be a coheir sequence of some global coheir of $\operatorname{tp}(g / G)$ where $g$ has idempotent orbit. Let $\bar{h} \in g^{\omega}$ be such that $h_{i} \in \operatorname{fp}\left(\overleftarrow{g}_{\left\ulcorner_{i}\right.}\right)$ for some finite non-empty $I_{i} \subseteq \omega$ such that $I_{i}<I_{i+1}$. Then $\bar{h} \equiv_{G} \bar{g}$.

Proof Write $n_{i}$ for the minimum of $I_{i}$. It suffices to prove that $h_{i} \equiv_{G, g_{\mid n_{i}}} g_{n_{i}}$. Note that the type $g \equiv_{G} x \beth_{G} g_{\mid n_{i}}$ is satisfied both by $h_{i}$ and $g_{n_{i}}$, hence the claim follows by stationarity.

Write $\mathrm{fp}(\bar{a})_{n}$ for the $n$-uniform hypergraph with vertex set $\mathrm{fp}(\bar{a})$ and as edges those sets $\left\{h_{1}, \ldots, h_{n}\right\}$ such that $h_{i} \in \operatorname{fp}\left(a_{\left\lceil I_{i}\right.}\right)$ for some finite sets $I_{1}<\cdots<I_{n}$.

5.3 Milliken-Taylor Theorem Let $<$ be a relation on $G$ that makes it $<-$ closed and $<-$ covered. Then for every positive integer $n$ and every finite coloring of the complete n-uniform hypergraph with vertex set $G$ there is $a<$-chain $\bar{a}$ such that $\mathrm{fp}(\bar{a})_{n}$ is monochromatic.

Proof Given a coheir sequence $\bar{g}$ as in the proof of Theorem 5.1 we want to define $\bar{a} \in G^{\omega}$ such that $\mathrm{fp}(\bar{a})_{n}$ is monochromatic. By the proposition above, $\mathrm{fp}\left(\overleftarrow{g}_{\mid i}\right)_{n}$ is monochromatic for every $i \geq n$. As in the proof of Theorem 5.1, we define by induction $\bar{a} \in G^{\omega}$ in such a way that $\operatorname{fp}\left(a_{\mid i}, \overleftarrow{g}_{\mid n}\right)_{n}$ is a finite monochromatic subgraph of $G$. 


\section{The Hales-Jewett theorem}

The Hales-Jewett theorem is a purely combinatorial statement that implies the van der Waerden theorem. The original proof by Alfred Hales and Robert Jewett is combinatorial [9]. An alternative proof, also combinatorial, is due by Saharon Shelah [16]. Our proof is similar to the proof by Andreas Blass in [4] (based on ideas from [3]), but we use saturated models where he uses Stone-Čech compactification. We present three versions of the main theorem.

First we prove an abstract algebraic version due to Sabine Koppelberg [11] which is easier to state and to prove (this version comes in two variants). The classical version follows easily from the algebraic one.

We work with the same notation as in Section 4. We say that an element $c$ is left-minimal (w.r.t. $\mathcal{A}$ ) if $c \in \mathcal{A} \cdot G g$ for every $g \in \mathcal{A} \cdot{ }_{G} c$.

6.1 Proposition Assume $I_{G}$ is 1-stationary. Let $\mathcal{A}$ be idempotent and type-definable over $G$. Then $\mathcal{A}$ contains a left-minimal element $c$ with idempotent orbit.

Proof Construct by induction a chain of type-definable idempotent sets $\mathcal{B}_{\alpha} \subseteq \mathcal{A}$ and elements $b_{\alpha} \in \mathcal{B}_{\alpha}$ such that $\mathcal{B}_{0}=\mathcal{A}$ and $\mathcal{B}_{\alpha+1}=\mathcal{A} \cdot{ }_{G} b_{\alpha}$. For $\alpha$ limit take the intersection. By idempotency of $\mathcal{A}$, it is straightforward to check that $\mathcal{B}_{\alpha+1} \subseteq \mathcal{B}_{\alpha}$. The sets $\mathcal{B}_{\alpha}$ are type-definable and idempotent by 4.1 and 4.3. For $\alpha$ limit $\mathcal{B}_{\alpha}$ is non-empty by compactness, as it is intersection of a chain of closed sets.

For some $\alpha$ we cannot properly extend this construction. For this $\alpha$, for every $c, g \in \mathcal{B}_{\alpha}$ we have $\mathcal{A} \cdot{ }_{G} c=\mathcal{B}_{\alpha}=\mathcal{A} \cdot G g$. Hence every $c \in \mathcal{B}_{\alpha}$ is left-minimal. As $\mathcal{B}_{\alpha}$ is idempotent, by Corollary 4.7 there is some $c \in \mathcal{B}_{\alpha}$ with idempotent orbit.

6.2 Proposition Assume $I_{G}$ is 1-stationary. Let $\mathcal{A}$ be idempotent and type-definable over $G$. Let $c_{G}$ be idempotent and such that $c \cdot G \mathcal{A}, \mathcal{A} \cdot G \subset \subseteq \mathcal{A}$. Then

1. $C \cdot G \mathcal{A} \cdot G C$ contains some $g$ with idempotent orbit;

2. if moreover $c$ is left-minimal, then $c \equiv_{G} g$ for every $g$ as in 1 .

Note, parenthetically, that the set in 1 may not be type-definable, therefore Corollary 4.7 does not apply directly and we need an indirect argument.

Proof 1. From $c \cdot{ }_{G} \mathcal{A} \subseteq \mathcal{A}$ we obtain that $\mathcal{A}{ }_{G}{ }_{G} c$ is idempotent. As it is also typedefinable, $\mathcal{A} \cdot{ }_{G} c$ contains a $b$ with idempotent orbit by Corollary 4.7. There is an $a \in \mathcal{A}$ such that $b_{G}=a \cdot{ }_{G} c$, then $b \cdot{ }_{G} c=b_{G}$. From this we obtain that $c \cdot{ }_{G} b$ is idempotent and contained in $c \cdot{ }_{G} \mathcal{A} \cdot{ }_{G} c$.

2. From $g \in c \cdot{ }_{G} \mathcal{A} \cdot{ }_{G} c$ and the idempotency of $c_{G}$ we obtain $g_{G}=c \cdot G g$. As $g \in \mathcal{A} \cdot{ }_{G} c$, from the left-minimality of $c_{G}$ we obtain $c \in \mathcal{A} \cdot G g$. Hence $c_{G}=c \cdot G g$, by the idempotency of $g_{G}$. Therefore $c_{G}=g_{G}$, which proves 2 .

The following is a technical lemma that is required in many proofs below. 
6.3 Proposition Assume $\mathcal{I}_{G}$ is 1-stationary. Let $\sigma: \mathcal{G} \rightarrow \mathcal{G}$ be a semigroup homomorphism definable over $G$. Then for every $a, b \in \mathcal{G}$

1. $\sigma\left[a_{G}\right]=(\sigma a)_{G}$

2. $\sigma[a \cdot G b]=\sigma a \cdot G \sigma b$.

Proof 1. As $a \equiv_{G} a^{\prime}$ implies $\sigma a \equiv_{G} \sigma a^{\prime}$, inclusion $\subseteq$ is clear. For the converse, note that the type $\exists y\left[\sigma y=x \wedge y \equiv_{G} a\right]$ is trivially realized by $\sigma a$. Therefore it is realized by all elements of $(\sigma a)_{G}$. Hence all elements of $(\sigma a)_{G}$ are the image of some element in $a_{G}$.

2. Let $a \equiv_{G} a^{\prime} I_{G} b^{\prime} \equiv_{G} b$. By Proposition 4.4 we have $\sigma[a \cdot G b]=\sigma\left[\left(a^{\prime} \cdot b^{\prime}\right)_{G}\right]$. Then it suffices to prove that $\sigma\left[\left(a^{\prime} \cdot b^{\prime}\right)_{G}\right] \subseteq \sigma a \cdot{ }_{G} \sigma b$, because by 1 and Proposition 4.4 both sides of the equality are orbits. As $\sigma$ preserves $I_{G}$ and orbits, we obtain that $\sigma\left(a^{\prime} \cdot b^{\prime}\right)$ is in $\sigma a \cdot G \sigma b$, as well as all other elements of $\sigma\left[\left(a^{\prime} \cdot b^{\prime}\right)_{G}\right]$.

6.4 Hales-Jewett Theorem (Koppelberg's version) Let $G$ be an infinite semigroup and let $C \subset G$ be a nice subsemigroup. Let $\Sigma$ be a finite set of retractions of $G$ onto $C$. Then, for every finite coloring of $C$, there is an $a \in G \backslash C$ such that $\{\sigma a: \sigma \in \Sigma\}$ is monochromatic.

Proof Let $G \preceq \mathcal{G}$. Here $\mathcal{G}$ is a monster model in a language that expands the natural one with a symbol for all subsets of $G$ and for every retraction in $\Sigma$. As observed in Remark 2.7, this makes $\mathcal{I}_{G}$ trivially 1-stationary. Let $\mathcal{C}$ be the definable set such that $C=G \cap \mathcal{C}$. By elementarity, $C$ is a nice subsemigroup of $\mathcal{G}$. The language contains also symbols for the retractions $\sigma: \mathcal{G} \rightarrow \mathcal{C}$.

By Proposition 6.1, there is a left-minimal $c \in \mathcal{C}$ with idempotent orbit.

By niceness, $G \backslash C$ and $c$ satisfy the assumptions of Proposition 6.2. Hence, by the first claim of that proposition, there is an idempotent $g \in c \cdot G(\mathcal{G} \backslash \mathcal{C}) \cdot{ }_{G} c$. In particular, $g \in \mathcal{G} \backslash \mathcal{C}$. Now apply the second claim of Proposition 6.3, with $\mathcal{C}$ for $\mathcal{A}$ to obtain $\sigma g \in C \cdot{ }_{G} \mathcal{C} \cdot{ }_{G} c$ for all $\sigma \in \Sigma$. As $\sigma g$ is also idempotent, we apply Proposition 6.2 to conclude that $\sigma g \equiv_{G} c$. In particular the set $\{\sigma g: \sigma \in \Sigma\}$ is monochromatic.

Though the element $g$ above need not belong to $G \backslash C$, by elementarity $G \backslash C$ contains some $a$ with the same property and this proves the theorem.

Finally we show how the classical Hales-Jewett theorem follows from its abstract version.

If $C$ and $X$ are two semigroups we denote by $C * X$ their free product. That is, $C * X$ contains finite sequences of elements of $C \cup X$, below called words, that alternate elements in $C$ with elements in $X$. The product of two words is obtained concatenating them and, when it applies, replacing two contiguous elements of the same semigroup by their product. Note that $C$ and $X$ are nice subsemigroups of $C * X$. When $X$ is the free semigroup generated by a variable $x$, we denote $C * X$ by $C[x]$. If $w(x)$ is an element of $C[x]$ and $a \in C$ we denote by $w(a)$ the result of 
replacing $x$ by $a$ in $w(x)$.

6.5 Hales-Jewett Theorem (classical version) Let $C$ be a semigroup generated by some finite set $A$. Let $x$ be a variable. Then for every finite coloring of $C[x]$ there is a $w(x) \in C[x] \backslash C$ such that $\{w(a): a \in A\}$ is monochromatic.

Proof Let $G=C[x]$. For every $a \in A$ the homomorphism $\sigma_{a}: w(x) \mapsto w(a)$ is a retraction of $G$ onto $C$. Hence we can apply the theorem above.

We conclude with a variant of Theorem 6.4 that applies to a broader class of semigroup homomorphisms. This result is not required for the following.

For $\Sigma$ a set of maps $\sigma: G \rightarrow C$ and $c \in C$ we define

$$
\Sigma^{-1}[c]=\bigcap_{\sigma \in \Sigma} \sigma^{-1}[c]
$$

Clearly, when the maps in $\Sigma$ are retractions, $\Sigma^{-1}[c]$ is non-empty for all $c \in C$ because it contains at least $c$.

6.6 Hales-Jewett Theorem (yet another variant) Let $\mathrm{C}$ be a semigroup and let $\Sigma$ be a finite set of homomorphisms $\sigma: G \rightarrow C$ such that $\Sigma^{-1}[c]$ is non-empty for all $c \in C$. Then, for every finite coloring of $C$, there is a $g \in G$ such that the set $\{\sigma g: \sigma \in \Sigma\}$ is monochromatic.

Proof Let $G * C$ be the free product of the two semigroups. Any homomorphism $\sigma: G \rightarrow C$ extends canonically to a retraction of $G * C$ onto $C$. The elements of $G$ that occur in a word are replaced by their image under $\sigma$, finally the elements in the resulting sequence are multiplied. This extension is denoted by the same symbol $\sigma$.

Apply Theorem 6.4 to obtain some $w \in G * C$ such that $\{\sigma w: \sigma \in \Sigma\}$ is monochromatic. Suppose $w=c_{0} \cdot g_{0} \cdots \cdots c_{n} \cdot g_{n}$ for some $g_{i} \in G$ and $c_{i} \in C$, where one or both of $c_{0}$ or $g_{n}$ could be absent. Pick some $h_{i} \in \Sigma^{-1}\left[c_{i}\right]$ and let $g=$ $h_{0} \cdot g_{0} \cdots \cdots h_{n} \cdot g_{n}$. Then $\{\sigma g: \sigma \in \Sigma\}$ is monochromatic as required to complete the proof.

\section{Carlson's theorem}

This section is devoted to the following lemma and some of its consequences.

7.1 Lemma Let $\Sigma$ be a finite set of retractions of $G$ onto a nice subsemigroup $C$. Let $<$ be a relation on $G$ that makes it $<-$ closed and $<$-covered by $G \backslash C$. Then, for every finite coloring of $G$, there is $a<-$-chain $\bar{a} \in(G \backslash C)^{\omega}$ such that $\mathrm{fp}^{\Sigma} \bar{a} \backslash C$ is monochromatic.

Proof The models $\mathcal{G}$ and $C$ are as in the proof of Theorem 6.4. The language is the same with $<$ included. Let $\mathcal{B}=\{g \in \mathcal{G} \backslash C: G<g\}$. By Proposition 6.1 there is some left-minimal $c \in \mathcal{C}$ with idempotent orbit. As $G$ is $<$-covered by $G \backslash C$, the set $\mathcal{B}$ is non-empty. As $G$ is $\longleftarrow$-closed and $C$ is nice, $\mathcal{B}$ and $c$ satisfy the assumptions of Proposition 6.2. Then, $c \cdot G \mathcal{B} \cdot G c$ contains some $g_{0}$ with idempotent orbit. By 
Proposition 6.3, we obtain that $\sigma g_{0} \in C \cdot{ }_{G} \mathcal{C} \cdot{ }_{G} c$ for all $\sigma \in \Sigma$. As $\left(\sigma g_{0}\right)_{G}$ is also idempotent, we apply the second claim of Proposition 6.3, with $\mathcal{C}$ for $\mathcal{A}$ to conclude that $\sigma g_{0} \equiv_{G} c$ for all $\sigma \in \Sigma$. Now, let $\bar{g}$ be a coheir sequence as in Theorem 5.1, and assume the notation thereof. As $g_{0} \in c \cdot G \mathcal{B} \cdot G c$ then $c \cdot G g_{0}=g_{0} \cdot{ }_{G} c=\left(g_{0}\right)_{G}$. Hence $h \equiv_{G} g_{0}$ for all $i$ and all $h \in \operatorname{fp} \overleftarrow{g}_{\mid i} \backslash C$. In particular all these $h$ have the same color, say color 1 . Now, we can use the sequence $\bar{g}$ to define $\bar{a} \in(G \backslash C)^{\omega}$ such that all elements of $\operatorname{fp}^{\Sigma} \bar{a} \backslash C$ have color 1 by reasoning as in the proof of Theorem 5.1.

Carlson's theorem is a result that combines the theorems of Hindman and HalesJewett and has a number of important consequences. We refer the reader to [7] for a discussion of some of these consequences. The definitions in Example 1.1 will help matching the notation.

We first present a Koppelberg-style version of the theorem. It is obtained from the lemma above applying a suitable coding.

7.2 Carlson Theorem (à la Koppelberg) Let $\Sigma$ be a finite set of retractions of $G$ onto a nice subsemigroup $C$. Let $\bar{s} \in(G \backslash C)^{\omega}$. Then for every finite coloring of $G$, there is an increasing sequence of positive integers $\left\langle n_{i}: i<\omega\right\rangle$ and some $a_{i} \in \mathrm{fp}^{\Sigma_{S_{\Gamma}}} n_{\left.n_{i}, n_{i+1}\right)} \backslash C$ such that $\operatorname{fp}^{\Sigma} \bar{a} \backslash C$ is monochromatic.

Proof Let $G_{*}$ be the free semigroup generated by the alphabet

$$
\left\{\langle\sigma, g\rangle: \sigma \in \Sigma \cup\left\{\operatorname{id}_{G}\right\}, g \in G \backslash C\right\} .
$$

The semigroup $C_{*}$ is defined as $G_{*}$, only $\sigma$ is restricted to range over $\Sigma$. Clearly $C_{*}$ is a nice subsemigroup of $G_{*}$. We associate to each $\sigma \in \Sigma$ the endomorphism of $G_{*}$ that substitutes $\sigma$ for every occurrence of $\operatorname{id}_{G}$ in a word. These maps, which we denote by $\sigma_{*}$, are retractions of $G_{*}$ onto $C_{*}$.

If $g_{*} \in G_{*}$ has the form $\left\langle\sigma_{1}, g_{1}\right\rangle \cdots\left\langle\sigma_{n}, g_{n}\right\rangle$ we call $\sigma_{1} g_{1} \cdots \sigma_{n} g_{n} \in G$ the evaluation of $g_{*}$. We denote the evaluation by eval $\left(g_{*}\right)$. As $\tau \sigma=\sigma$ for every $\tau, \sigma \in \Sigma$, we have that eval $\left(\sigma_{*} g_{*}\right)=\sigma \operatorname{eval}\left(g_{*}\right)$. The evaluation of $g_{*} \in C_{*}$ belongs to $C$ and, as $C$ is nice, the evaluation of $g_{*} \in G_{*} \backslash C_{*}$ belongs to $G \backslash C$.

We color each element of $G_{*}$ with the color of its evaluation.

We define the relation $<$ on $G_{*}$. First, we need to define the well-formed elements of $G_{*}$. These are elements of the form $\left\langle\sigma_{1}, s_{i_{1}}\right\rangle \cdots\left\langle\sigma_{n}, s_{i_{n}}\right\rangle$ for some $i_{1}<\cdots<i_{n}$. Now, for $h_{*}, g_{*} \in G_{*}$ we define $h_{*}<g_{*}$ if one of the following holds

1. $h_{*}$ is not well-formed while $g_{*}$ is;

2. the product (i.e., concatenation) $h_{*} g_{*}$ is well-formed.

It is immediate to verify that $<$ is $G_{*}$ is $<$-closed and $<$-covered by $G_{*} \backslash C_{*}$. Therefore by Lemma 7.1 there is a $<$-chain $\bar{a}_{*} \in\left(G_{*} \backslash C_{*}\right)^{\omega}$ such that $\mathrm{fp}^{\Sigma} \bar{a}_{*} \backslash C_{*}$ is monochromatic. We can assume that all elements of $\bar{a}_{*}$ are well-formed (only the first element might be ill-formed, but we can drop it). Then the sequence $\left\langle\operatorname{eval}\left(a_{i *}\right): i \in \omega\right\rangle$ is as required by the lemma. 
From the algebraic version of Carlson's theorem we obtain the classical one in the same way as for the Hales-Jewett theorem (Theorem 6.5), which we refer to for the notation.

7.3 Corollary (Carlson's theorem, classical version) Let $C$ be a semigroup generated by some finite set $A$. Let $x$ be a variable. Let $\bar{s} \in(C[x] \backslash C)^{\omega}$. Let $\Sigma$ contain, for every $a \in A$, the function $w(x) \mapsto w(a)$. Then, for every finite coloring of $C[x]$, there is an increasing sequence of positive integers $\left\langle n_{i}: i<\omega\right\rangle$ and some $a_{i} \in \mathrm{fp}^{\Sigma s_{\Gamma\left[n_{i}, n_{i+1}\right)} \backslash C \text { such }}$ that $\operatorname{fp}^{\Sigma} \bar{a} \backslash C$ is monochromatic (with the terminology of Example 1.1, $\bar{a}$ is an extracted variable sequence of $\bar{s}$ ).

\section{Gowers's partition theorem}

The following is similar to Lemma 7.1 but here $\Sigma$ contains compositions of homomorphisms.

8.1 Lemma For $0<i<n$, let $G_{i}$ be a nice subsemigroup of $G_{i+1}$ and let $\sigma_{i}: G_{i+1} \rightarrow G_{i}$ be homomorphisms. Let $<$ be a relation on $G_{n}$ that makes it $<$-closed and $<$-covered by $G_{n} \backslash G_{n-1}$. Finally, let $\Sigma=\left\{\sigma_{i} \circ \cdots \circ \sigma_{n-1}: 0<i<n\right\}$. Then, for every finite coloring of $G_{n}$, there is $a<$-chain $\bar{a} \in\left(G_{n} \backslash G_{n-1}\right)^{\omega}$ such that $\mathrm{fp}^{\Sigma} \bar{a} \backslash G_{n-1}$ is monochromatic.

Proof For convenience, we let $i$ run from 0 , hence we agree that $\sigma_{0}: G_{1} \rightarrow G_{0}=G_{1}$ is the identity. Let $\mathcal{B}_{n}=\left\{b \in \mathcal{G}_{n} \backslash \mathcal{G}_{n-1}: G_{n}<b\right\}$ and $\mathcal{B}_{i}=\sigma_{i}\left[\mathcal{B}_{i+1}\right]$. Note that the $\mathcal{B}_{i}$ are non-empty because $G_{n}$ is <-covered by $G_{n} \backslash G_{n-1}$. Also, as $\mathcal{G}_{i}$ is a nice subsemigroup of $\mathcal{G}_{i+1}$, we have that $\mathcal{B}_{i} \cdot{ }^{\cdot G} \mathcal{B}_{i+1}, \mathcal{B}_{i+1} \cdot G \mathcal{B}_{i} \subseteq \mathcal{B}_{i+1}$.

We claim there is some $b_{n} \in \mathcal{B}_{n}$ with idempotent orbit such that, if we define $b_{i}=\sigma_{i} b_{i+1}$ for $0 \leq i<n$, the following holds

$$
b_{n} \cdot G b_{i}=b_{i} \cdot G b_{n}=\left(b_{n}\right)_{G} .
$$

Note that these equalities may be replaced by

$$
\sharp_{i} \quad b_{i} \cdot G b_{i+1}=b_{i+1} \cdot G b_{i}=\left(b_{i+1}\right)_{G} \text {. }
$$

Let $b_{0}=b_{1}$ be any element of $\mathcal{B}_{0}$ with idempotent orbit. We assume as induction hypothesis that we have $b_{i} \in \mathcal{B}_{i}$ for $i \leq k$, with idempotent orbits, such that $b_{i}=$ $\sigma_{i} b_{i+1}$ and $\sharp_{i}$ hold for all $i<k$. We show how to find $b_{k+1}$.

We prove that $b_{k}$ and the set $\mathcal{B}_{k+1} \cap \sigma_{k}^{-1}\left[b_{k}\right]$, which below we denote by $\mathcal{A}$ for short, satisfy the assumptions of Proposition 6.2. The proof of the idempotency of $\mathcal{A}$ is left to the reader. We prove that $b_{k} \cdot{ }_{G} \mathcal{A} \subseteq \mathcal{A}$, the proof of $\mathcal{A} \cdot{ }_{G} b_{k} \subseteq \mathcal{A}$ is similar. As $b_{k} \cdot G \mathcal{B}_{k+1} \subseteq \mathcal{B}_{k+1}$ by nicety, it suffices to prove that $b_{k} \cdot G \sigma_{k}^{-1}\left[b_{k}\right]$ is contained in $\sigma_{k}^{-1}\left[b_{k}\right]$. This latter inclusion holds because, by the induction hypothesis,

$$
\sigma_{k}\left[b_{k} \cdot G \sigma_{k}^{-1}\left[b_{k}\right]\right]=\sigma_{k}\left[b_{k}\right] \cdot G b_{k}=b_{k-1} \cdot G b_{k}=\left(b_{k}\right)_{G} .
$$

Now we apply Proposition 6.2 to find an idempotent $b_{k+1} \in b_{k} \cdot{ }_{G} \mathcal{A} \cdot{ }_{G} b_{k}$. Therefore $\sharp_{k}$ is satisfied. Moreover $\sigma_{k} b_{k+1} \in\left(b_{k}\right)_{G}$ by Proposition 6.3, hence we can assume 
$b_{k}=\sigma_{k} b_{k+1}$ as claimed above.

Finally, as in the proof of Theorem 5.1, the required chain $\bar{a}$ is obtained from a coheir sequence of a global coheir of $\operatorname{tp}\left(b_{n} / G\right)$.

8.2 Remark The lemma above continues to hold, with essentially the same proof, if for $\Sigma$ we take a set of the form

where

$$
\Sigma=\bigcup_{i=1}^{n-1} \Sigma_{i} \circ \cdots \circ \Sigma_{n-1}
$$

$$
\Sigma_{i} \circ \cdots \circ \Sigma_{n-1}=\left\{\sigma_{i} \circ \cdots \circ \sigma_{n-1}: \sigma_{i} \in \Sigma_{i}, \ldots, \sigma_{n-1} \in \Sigma_{n-1}\right\}
$$

and where $\Sigma_{i}$ are some finite sets of homomorphisms $G_{i+1} \rightarrow G_{i}$ such that for every $g \in G_{i}$ the set $\Sigma_{i}^{-1}[g]$ is non-empty.

Let $G_{i}$ be the set of functions $a: \omega \rightarrow\{0, \ldots, i\}$ with finite support that is, the set $\operatorname{supp}(a)=\{x \in \omega: a x \neq 0\}$ is finite. We introduce a semigroup operation on $G_{i}$ by defining $(a \cdot b) x=\max \{a x, b x\}$. This makes $G_{i}$ a nice subsemigroup of $G_{i+1}$.

8.3 Corollary (Gowers Partition Theorem) With $G_{i}$ as above, let $\sigma_{i}: G_{i+1} \rightarrow G_{i}$ be homomorphisms and let $\Sigma$ be as in Lemma 8.1. Then for every finite coloring of $G_{n}$ there is an $\bar{a} \in\left(G_{n} \backslash G_{n-1}\right)^{\omega}$ such that $\mathrm{fp}^{\Sigma} \bar{a} \backslash G_{n-1}$ is monochromatic and $\operatorname{supp}\left(a_{i}\right)<\operatorname{supp}\left(a_{i+1}\right)$.

The homomorphisms $\sigma_{i}$ usually considered in the literature are so-called tetris operations i.e. $\left(\sigma_{i} a\right) x=\max \{a x-1,0\}$, or generalizations thereof. However the theorem is more general.

Proof Let $<$ be the relation $\operatorname{supp}(a)<\operatorname{supp}(b)$ and apply Theorem 8.1.

\section{References}

[1] Claudio Agostini and Eugenio Colla, Partition regularity for semigroups. In preparation.

[2] Uri Andrews, Gabriel Conant, and Isaac Goldbring, Definable sets containing productsets in expansions of groups, J. Group Theory 22 (2019).

[3] Vitaly Bergelson, Andreas Blass, and Neil Hindman, Partition theorems for spaces of variable words, Proc. London Math. Soc. (3) 68 (1994), no. 3, 449-476.

[4] Andreas Blass, Ultrafilters: where topological dynamics = algebra $=$ combinatorics, Topology Proc. 18 (1993), 33-56. Also arXiv:math/9309208.

[5] Mauro Di Nasso, Hypernatural numbers as ultrafilters, Nonstandard analysis for the working mathematician, Springer, Dordrecht, 2015, pp. 443-474.

[6] Mauro Di Nasso, Isaac Goldbring, and Martino Lupini, Nonstandard Methods in Ramsey Theory and Combinatorial Number Theory (2018). ArXiv:1709.04076. 
[7] Pandelis Dodos and Vassilis Kanellopoulos, Ramsey theory for product spaces, Mathematical Surveys and Monographs, vol. 212, American Mathematical Society, 2016.

[8] W. T. Gowers, Lipschitz functions on classical spaces, European J. Combin. 13 (1992), no. 3, 141-151.

[9] A. W. Hales and R. I. Jewett, Regularity and positional games, Trans. Amer. Math. Soc. 106 (1963), 222-229.

[10] Neil Hindman, Finite sums from sequences within cells of a partition of N, J. Combinatorial Theory Ser. A 17 (1974), 1-11.

[11] Sabine Koppelberg, The Hales-Jewett theorem via retractions, Proceedings of the 18th Summer Conference on Topology and its Applications, 2004, pp. 595-601.

[12] Martino Lupini, Actions on semigroups and an infinitary Gowers-Hales-Jewett Ramsey theorem, Trans. Amer. Math. Soc. 371 (2019).

[13] Keith R. Milliken, Ramsey's theorem with sums or unions, J. Combinatorial Theory Ser. A 18 (1975), 276-290.

[14] Ludomir Newelski, Topological dynamics of definable group actions, J. Symbolic Logic 74 (2009), no. 1, 50-72.

[15] __ , Topological dynamics of stable groups, J. Symb. Log. 79 (2014), no. 4, 11991223.

[16] Saharon Shelah, Primitive recursive bounds for van der Waerden numbers, J. Amer. Math. Soc. 1 (1988), no. 3, 683-697.

[17] Alan D. Taylor, A canonical partition relation for finite subsets of $\omega$, J. Combinatorial Theory Ser. A 21 (1976), no. 2, 137-146.

[18] Katrin Tent and Martin Ziegler, A course in model theory, Lecture Notes in Logic, vol. 40, Association for Symbolic Logic, Cambridge University Press, 2012.

[19] Domenico Zambella, A crèche course in model theory, AMS Open Math Notes, 2018. (The link points to the github version). 